\title{
Evaluation of the diagnostic efficiency of systemic immune-inflammation index in prostate biopsy
}

\author{
๑Mehmet Demir, @İsmail Yağmur, @Eyyup Sabri Pelit, @Bülent Katı, @İbrahim Halil Albayrak, \\ ๑DHalil Çiftçi \\ Harran University, Department of Urology, Şanlıurfa, Turkey
}

Cite this article as: Demir M, Yağmur İ, Pelit ES, Katı B, Albayrak İH, Çiftçi H. Evaluation of the diagnostic efficiency of systemic immuneinflammation index in prostate biopsy. J Health Sci Med 2021; 4(6): 897-900.

\begin{abstract}
Aim: This study aimed to evaluate the diagnostic efficacy of systemic immune-inflammation index (SII) in patients with prostate cancer ( $\mathrm{PCa}$ ) who have undergone prostate biopsy and have a prostate-specific antigen (PSA) value of $4-10 \mathrm{ng} / \mathrm{ml}$.

Material and Method: In this study we included patients with a PSA value of 4-10 ng/mL, who underwent transrectal ultrasound-guided 12-core prostate biopsy between January 2010 and March 2021. The patients were divided into two groups as those with and without cancer. Those with cancer were divided into two groups as low grade (grade 1 and 2) and high grade (grade 3-5) according to the International Society of Urologic Pathologists (ISUP) grades. PSA, neutrophil-lymphocyte ratio (NLR), prostate volume, platelet-to-lymphocyte ratio (PLR) and SII were compared between the groups.

Results: The mean age of the 182 patients included in the study was $63.4 \pm 8.0$ years, and the mean PSA value was $6.69 \pm 2.45 \mathrm{ng} /$ $\mathrm{mL}$. Benign prostate disease were detected in $142(78 \%)$ of the patients and PCa in $40(22 \%)$ patients. PSA, PLR, NLR and SII did not differ in those with cancer. Low ISUP grade cancer was detected in $80 \%$ of the patients and high ISUP grade cancer in 20\%. PSA and PLR values were significantly higher in patients with high ISUP grade PCa compared to those with low ISUP grade $(\mathrm{p}<0.05)$. There was no difference in SII values between the two groups. PSA, PLR and SII values of patients with high ISUP grade PCa were significantly higher than those without cancer $(\mathrm{p}<0.05)$.

Conclusions: SII and PLR, which can be easily calculated from peripheral blood, can be an effective predictor of pre-biopsy high-grade ISUP PCa.
\end{abstract}

Keywords: Prostate cancer, diagnosis, systemic immune-inflammation index, platelet-to-lymphocyte ratio, biopsy

\section{INTRODUCTION}

Prostate cancer $(\mathrm{PCa})$ is the most common cancer in men following lung cancer. Mortality rates vary worldwide, with the highest being in the African descent and lowest in Asian (1). The use of serum prostate-specific antigen (PSA) has become widespread due to the increasing elderly population. Accordingly, prostate biopsy rates have increased significantly. Although the PSA value to predict cancer remains unknown, $4 \mathrm{ng} / \mathrm{mL}$ is used as a cutoff value for prostate biopsy (2). Since PSA can increase in benign prostate diseases, it is not an ideal biomarker for PCa (3). PCa was detected in approximately $25 \%$ of prostate biopsies performed with a cut-off value of 4.0 $\mathrm{ng} / \mathrm{mL}$; however, approximately $50 \%$ of the detected cancers were clinically insignificant (4). PSA density and free PSA/total PSA are used in the differentiation of cancer and benign diseases; however, their sensitivity

and specificity are low (5). Therefore, there is a need for effective biomarkers to detect clinically important cancer.

Inflammation is an important predictor of tumour invasion and progression (6) and plays a role in $\mathrm{PCa}$ development (7). Therefore, inflammation parameters, such as neutrophil-to-lymphocyte ratio (NLR) and platelet-to-lymphocyte ratio (PLR) are used as tumour markers. Systemic immune-inflammation index (SII) is a new inflammatory marker calculated from lymphocytes, neutrophils and platelets and is used in cancer diagnosis and prognosis (8). In this study, we aimed to evaluate the effectiveness of NLR, PLR and SII in distinguishing PCa and clinically important cancer in patients with a PSA value of 4-10 $\mathrm{ng} / \mathrm{mL}$ and who underwent biopsy for PCa screening. 


\section{MATERIAL AND METHOD}

The study was carried out with the permission of Harran University Clinical Researchs Ethics Committee (Date: 12.04.2021, Decision No: HRU/21.08.07). All procedures were carried out in accordance with the ethical rules and the principles of the Declaration of Helsinki.

The data of patients who underwent prostate biopsy for prostate cancer screening between January 2010 and March 2021 were retrospectively reviewed. Patients with a total PSA value of $4-10 \mathrm{ng} / \mathrm{mL}$ were included in the study. Patients who had previously undergone prostate biopsy and those with suspected cancer during the rectal examination were not included in the study. Before the biopsy, urine culture and complete blood count were performed. Appropriate treatment was applied to patients with infection in the analysis results, and biopsy was performed after their cultures were negative. All patients received antibiotic prophylaxis before the biopsy. All patients underwent a 12-core prostate biopsy using an 18 gauge Tru-Cut needle accompanied by transrectal ultrasound. From the complete blood count results, NLR, PLR and SII (neutrophil count $x$ platelet count / lymphocyte count) were calculated. Prostate volume was calculated according to the formula $0.52 \times$ transverse $\times$ anteroposterior $\times$ transverse $\times$ longitudinal diameters. The patients were divided into two groups those with and those without PCa. Those with PCa were divided into two groups low ISUP grade (grade 1 and 2) and high ISUP grade (grade 3-5) according to the International Society of Urological Pathology 2014. Age, PSA, prostate volume, lymphocyte, neutrophil, platelet, NLR, PLR and SII were compared between the groups.

\section{Statistical Analysis}

Mean, lowest and highest median values, standard deviation, ratio values and frequency were used in the descriptive statistics of the data. Kolmogorov-Smirnov test was used to measure the distribution of variables. Quantitative independent data was analyzed using independent samples t-test and Mann-Whitney $U$ test. SPSS 27.0 was used for statistical analyses.

\section{RESULTS}

The mean age of the 182 men included in the study was $63.4 \pm 8.0$ years, and the mean PSA value was $6.69 \pm 2.45$ ng/mL (Table 1).

Benign prostate diseases were detected in $142(78 \%)$ patients and $\mathrm{PCa}$ in $40(22 \%)$ patients. There was no significant difference $(\mathrm{p}>0.05)$ in age, prostate volume, PSA value, lymphocyte value, neutrophil value, NLR, PLR and SII ratio between the groups with and without PCa. Platelet value was significantly higher $(\mathrm{p}<0.05)$ in the group with PCa than in the group without PCa (Table 2).
Low ISUP grade cancers were detected in $80 \%$ of the patients and high ISUP grade cancers in $20 \%$ of the patients. Patients' age, lymphocyte value, neutrophil value, NLR ratio and SII ratio did not differ between the low ISUP grades and high ISUP grades groups ( $p>0.05$ ). PSA value, platelet value and PLR were significantly higher $(\mathrm{p}<0.05)$ in the group with high ISUP grades compared to the group with low ISUP grades (Table 3). PSA, PLR and SII were significantly higher in patients with high ISUP grade PCa compared with those without cancer $(\mathrm{p}<0.05)$.

\begin{tabular}{|c|c|c|c|}
\hline & Min-Max & Median & $\operatorname{Mean} \pm \mathbf{s d} / \mathbf{n}-\%$ \\
\hline Age & $44.0-78.0$ & 63.0 & $63.4 \pm 8.0$ \\
\hline PSA & $4.00-10.00$ & 6.294 & $6.69 \pm 2.45$ \\
\hline Lymphocyte & $0.40-4.40$ & 1.90 & $2.05 \pm 0.79$ \\
\hline Neutrophil & $1.50-45.00$ & 5.25 & $5.69 \pm 3.75$ \\
\hline Platelet & $111.0-446.0$ & 267.5 & $267.8 \pm 66.0$ \\
\hline NLR & $0.71-15.52$ & 2.64 & $3.16 \pm 2.24$ \\
\hline PLR & $47.1-757.5$ & 129.6 & $148.6 \pm 82.9$ \\
\hline SII & $160.3-4851.7$ & 698.6 & $843.2 \pm 656.4$ \\
\hline \multirow{5}{*}{ ISUP grade } & I & 23 & $57.5 \%$ \\
\hline & II & 9 & $22.5 \%$ \\
\hline & III & 3 & $7.5 \%$ \\
\hline & IV & 3 & $7.5 \%$ \\
\hline & V & 2 & $5.0 \%$ \\
\hline
\end{tabular}

\begin{tabular}{|c|c|c|c|c|c|}
\hline & \multicolumn{2}{|c|}{ No cancer $n: 142$} & \multicolumn{2}{|c|}{ Cancer n:40 } & \multirow{2}{*}{$\mathbf{p}$} \\
\hline & Mean \pm sd & Median & Mean \pm sd & Median & \\
\hline Age & $63.3 \pm 8.0$ & 63.0 & $63.4 \pm 83.3$ & 66.0 & $0.986^{\mathrm{t}}$ \\
\hline PSA & $6.61 \pm 2.62$ & 6.24 & $6.93 \pm 1.91$ & 6.50 & $0.226^{\mathrm{m}}$ \\
\hline $\begin{array}{l}\text { Prostate } \\
\text { volume (mL) }\end{array}$ & $50.8 \pm 29.2$ & 48.00 & $47.7 \pm 16.9$ & 46.00 & $0.08^{\mathrm{m}}$ \\
\hline Lymphocyte & $2.00 \pm 0.67$ & 1.90 & $2.19 \pm 0.85$ & 2.10 & $0.080^{\mathrm{m}}$ \\
\hline Neutrophil & $5.72 \pm 4.21$ & 5.10 & $5.62 \pm 2.01$ & 5.60 & $0.416^{\mathrm{m}}$ \\
\hline Platelet & $253.1 \pm 62.0$ & 255.5 & $308.9 \pm 59.9$ & 296.5 & $0.000^{m}$ \\
\hline NLR & $3.11 \pm 1.91$ & 2.72 & $3.29 \pm 3.00$ & 2.27 & $0.253^{\mathrm{m}}$ \\
\hline PLR & $138.4 \pm 52.9$ & 126.0 & $177.2 \pm 132.2$ & 144.8 & $0.146^{\mathrm{m}}$ \\
\hline SII & $780.4 \pm 498.0$ & 714.6 & $1019.3 \pm 958.9$ & 694.3 & $0.376^{\mathrm{m}}$ \\
\hline
\end{tabular}

Table 3. Comparison of patients according to ISUP grades

\begin{tabular}{|c|c|c|c|c|c|}
\hline & \multicolumn{2}{|c|}{ ISUP Grade I-II } & \multicolumn{2}{|c|}{ ISUP Grade III-IV-V } & \multirow{2}{*}{$\mathbf{p}$} \\
\hline & Mean \pm sd & Median & Mean \pm sd & Median & \\
\hline Age & $62.5 \pm 8.0$ & 64.5 & $66.9 \pm 8.9$ & 67.0 & $0.249^{\mathrm{m}}$ \\
\hline PSA & $6.55 \pm 1.74$ & 6.10 & $8.44 \pm 1.93$ & 9.20 & $0.016^{\mathrm{m}}$ \\
\hline Lymphocyte & $2.23 \pm 0.77$ & 2.20 & $2.04 \pm 1.17$ & 1.75 & $0.278^{\mathrm{m}}$ \\
\hline Neutrophil & $5.71 \pm 1.92$ & 5.60 & $5.26 \pm 2.43$ & 5.40 & $0.735^{\mathrm{m}}$ \\
\hline Platelet & $293.3 \pm 52.1$ & 286.0 & $371.0 \pm 49.5$ & 368.5 & $0.001^{\mathrm{m}}$ \\
\hline NLR & $3.16 \pm 2.72$ & 2.29 & $3.78 \pm 4.15$ & 2.10 & $0.685^{\mathrm{m}}$ \\
\hline PLR & $159.5 \pm 121.6$ & 130.0 & $248.0 \pm 157.6$ & 222.7 & $0.046^{\mathrm{m}}$ \\
\hline SII & $934.1 \pm 800.6$ & 667.3 & $1359.9 \pm 1455.4$ & 823.0 & $0.279^{\mathrm{m}}$ \\
\hline
\end{tabular}




\section{DISCUSSION}

Only half of the patients with PCa develop symptomatic disease throughout their lives (4). With the widespread use of PSA, a significant increase is observed in the diagnosis of clinically insignificant PCa $(9,10)$. While increased diagnosis leads to overtreatment, its impact on patients' overall quality of life is still uncertain (11). In patients with ISUP grade $1-2 \mathrm{PCa}$, the mortality rate is $7 \%$ in 15 years of follow-up, which is quite low (12). In addition, studies have shown that $10 \%$ of patients with clinically insignificant cancer develop severe depression as a side effect (13). Therefore, it is important to detect clinically significant PCa by avoiding overdiagnosis in patients with PSA values of $<10 \mathrm{ng} / \mathrm{ml}$ and no evidence of disease on rectal examination. In this study, cancer was detected in $22 \%$ of the patients who underwent prostate biopsy. Low ISUP grade (grade $1 ; 57.5 \%$, grade $2 ; 22.5 \%$ ) PCa was detected in $80 \%$ of these patients. These rates were similar to previous studies (4).

Inflammation is an important predictor of tumour invasion, progression and metastasis; additionally, it plays a role in PCa development $(7,14,15)$. Inflammatory markers, such as lymphocyte count, NLR, PLR and lymphocyte-to-monocyte ratio, are used in determining the diagnosis and prognosis of cancer due to their affordability and availability (16).

SII is calculated from the combination of NLR and the number of platelets, and studies emphasise that it may be a better marker than NLR (17). Recent studies have shown that high SII values in urological cancers are associated with poor prognosis $(8,18)$. Görgel et al. (19) reported that preoperative high SII value could be an independent prognostic factor in patients with muscle-invasive bladder tumour who underwent radical cystectomy, and cancer-specific survival was low in patients with SII $>843$. Another study evaluating patients who underwent nephroureterectomy for upper urinary tract ureteral cancers showed that high SII value is associated with high pathological stage and poor prognosis (20).

Studies examining the role of SII in PCa have been published in the recent years. Stangl-Kremser et al. (21) reported that SII may be a predictor of overall survival in patients with castration-resistant PCa. In the study by Rajwa et al. (22), in which the preoperative SII values of patients who underwent salvage radical prostatectomy were examined, it was shown that high SII value was a predictor for lymph node involvement and non-organ-confined disease. Another study published recently has investigated the effect of SII in cancer detection in patients who have undergone prostate biopsy. This study could not show an effect of SII in the distinction between PCa and benign prostate disease (23). In their study evaluating patients who underwent fission prostate biopsy, Sönmez et al. reported similar SII between benign prostatic diseases and low ISUP grades (grade 1 and 2) PCa. However, it was observed that SII was significantly higher in patients with high ISUP grades (grade 3-5) PCa (24). It has been reported that SII can be a predictor in detecting clinically important PCa. Although the SII value in patients with PCa in our study was higher than benign prostatic diseases, this difference was not statistically significant. Regarding the patients with PCa, there was no statistical difference between those with low ISUP grades and high ISUP grades. However, in patients with high ISUP grade PCa, the SII value was statistically significantly higher than benign prostatic diseases. Therefore, we think that SII can be a predictor in detecting clinically important PCa.

Patients with cancer usually have a hypercoagulable state. Platelets are an effective parameter in the progression of cancer and the prothrombotic state of the cancer patient. (25). Li et al. (15) reported that PLR value increases in patients with $\mathrm{PCa}$ and is an independent predictor of 3-year mortality. Adhyatma et al. (26) reported that PLR is a promising predictor of cancer in patients with suspected PCa. On the contrary, Murray et al. (23) found that PLR was similar between those with cancer and those with a benign prostate disease in patients who underwent prostate biopsy. In another study of metastatic PCa patients receiving hormonal therapy, NLR and PLR could not be associated with disease-specific survival, but platelet count was shown to be an independent prognostic factor (27). While the platelet value was significantly higher in patients with $\mathrm{PCa}$ in our study, PLR did not differ. However, in patients with high ISUP grades PCa, PLR and platelet values were significantly higher than those with both low ISUP grades PCa and benign prostate disease.

Our study includes some limitations. The first is the low number of patients. The reason for this is that patients with PSA $>10$ and patients with suspected cancer on rectal examination were excluded from the study. Another limitation is that the study is retrospective.

\section{CONCLUSION}

In PCa screening, ideal biomarkers that can differentiate benign and malignant tumours and detect clinically important cancer are needed to avoid unnecessary biopsies and overtreatment, especially in men with a PSA value in the gray zone $(4-10 \mathrm{ng} / \mathrm{mL})$ and no evidence of rectal examination. In our study, we showed that SII and PLR were higher in patients with higher ISUP grades than those with benign pathology. We think that SII and PLR, which can be easily calculated from peripheral blood, can be used as predictors of high ISUP grade PCa before performing a biopsy. For this, prospective studies with high numbers are needed. 


\section{ETHICAL DECLARATIONS}

Ethics Committee Approval: The study was carried out with the permission of Harran University Clinical Researchs Ethics Committee (Date: 12.04.2021, Decision No: HRU/21.08.07).

Informed Consent: Because the study was designed retrospectively, no written informed consent form was obtained from patients.

Referee Evaluation Process: Externally peer-reviewed.

Conflict of Interest Statement: The authors have no conflicts of interest to declare.

Financial Disclosure: The authors declared that this study has received no financial support.

Author Contributions: All of the authors declare that they have all participated in the design, execution, and analysis of the paper, and that they have approved the final version.

\section{REFERENCES}

1. Ferlay J, Soerjomataram I, Dikshit R, et al. Cancer incidence and mortality worldwide: sources, methods and major patterns in GLOBOCAN 2012. Int J Cancer 2015; 136: E359-86.

2. Canby-Hagino E, Hernandez J, Brand TC, Thompson I. Looking back at PCPT: looking forward to new paradigms in prostate cancer screening and prevention. Eur Urol 2007; 51: 27-33.

3. Vickers AJ, Sjoberg DD, Ulmert D, et al. Empirical estimates of prostate cancer overdiagnosis by age and prostate-specific antigen. BMC Med 2014; 12: 26.

4. Finne P, Fallah M, Hakama M, et al. Lead-time in the European Randomised Study of screening for prostate cancer. EurJ Cancer 2010; 46: 3102-8.

5. Catalona WJ, Southwick PC, Slawin KM, et al. Prospective evaluation of percent free PSA, PSA density and age specific PSA cut-offs for prostate cancer detection and staging. Urology 2000; 56: 255-60.

6. Bausch D, Pausch T, Krauss T, et al. Neutrophil granulocyte derived MMP-9 is a VEGF independent functional component of the angiogenic switchin pancreatic ductal adenocarcinoma. Angiogenesis 2011; 14: 235-43.

7. Yuksel OH, Urkmez A, Akan S, Yidirim C, Verit A. Predictive value of the platelet to lymphocyte ratio in the diagnosis of prostate cancer. Asian Pac J Cancer Prev 2015; 16: 6407-12.

8. Huang Y, Gao Y, Wu Y, Lin H. Prognostic value of systemic immune-inflammation index in patients with urologic cancers: a meta-analysis. Cancer Cell Int 2020; 20: 499.

9. Ilic D, Neuberger MM, Djulbegovic M, Dahm P. Screening for prostate cancer. Cochrane Database Syst Rev 2013; 31: CD004720.

10. Hayes JH, Barry MJ. Screening for prostate cancer with the prostate-specificantigen test: a review of current evidence. JAMA 2014; 311: 1143-9.

11. Booth N, Rissanen P, Tammela TL, Määttänen L, Taari K, Auvinen A. Health-related quality of life in the Finnish trial of screening for prostate cancer. Eur Urol 2014; 65: 39-47.

12. Albertsen PC. Observational studies and the natural history of screen-detected prostate cancer. Curr Opin Urol 2015; 25: 232-7.

13. Bellardita L, Villa S, Valdagni R. Living with untreated prostate cancer: predictors of quality of life. Curr Opin Urol 2014; 24: 311-7.

14. Ostan R, Lanzarini C, Pini E, et al. Inflammaging and cancer: a challenge for the Mediterranean diet. Nutrients 2015; 7: 2589-621.
15.Li F, Hu H, Gu S, Chen X, Sun Q. Platelet to lymphocyte ratio plays an important role in prostate cancer's diagnosis and prognosis. Int J Clin Exp Med 2015; 8: 11746-51.

16. Palacka P, Mego M, Mikulova T, et al. Systemic immuneinflammation index to predict survival in Caucasian patients with metastatic urothelial carcinoma. J Clin Oncol 2017; 35: e16015.

17. Aziz MH, Sideras K, Aziz NA, et al. The systemic-immuneinfammation index independently predicts survival and recurrence in resectable pancreatic cancer and its prognostic value depends on bilirubin levels: a retrospective multicenter cohort study. Ann Surg 2019; 270: 139-46.

18. Wang Q, Zhu SR, Huang XP, Liu XQ, Liu JB, Tian G. Prognostic value of systemic immune-inflammation index in patients with urinary system cancers: a meta-analysis. Eur Rev Med Pharmacol Sci 2021; 25: 1302-10.

19. Gorgel SN, Akin Y, Koc EM, Kose O, Ozcan S, Yilmaz Y. Retrospective study of systemic immune-inflammation index in muscle invasive bladder cancer: initial results of single centre. Int Urol Nephrol 2020; 52: 469-73.

20. Mori K, Resch I, Miura N, et al. Prognostic role of the systemic immune-inflammation index in upper tract urothelial carcinoma treated with radical nephroureterectomy: results from a large multicenter international collaboration. Cancer Immunol Immunother 2021; 70: 2641-50.

21.Stangl-Kremser J, Mari A, Suarez-Ibarrola R, et al. Development of a prognostic model for survival time prediction in castrationresistant prostate cancer patients. Urol Oncol 2020; 38: 600.

22. Rajwa P, Schuettfort VM, Quhal F, et al. Role of systemic immuneinflammation index in patients treated with salvage radical prostatectomy. World J Urol 2021; 17.

23. Murray NP, Fuentealba C, Salazar A, Reyes E. Platelet-tolymphocyte ratio and systemic immune-inflammation index versus circulating prostate cells to predict significant prostate cancer at first biopsy. Turk J Urol 2020; 46: 115-22.

24.Sonmez G, Demirtas T, Tombul ST, Akgun H, Demirtas A. Diagnostic efficiency of systemic immune-inflammation index in fusion prostate biopsy. Actas Urol Esp 2021; 45: 359-65.

25. Riedl J, Kaider A, Marosi C, et al. Decreased platelet reactivity in patients with cancer is associated with high risk of venous thromboembolism and poor prognosis. Thromb Haemost 2017; 117: 90-8.

26. Adhyatma KP, Warli SM. Diagnostic value of platelet-tolymphocyte ratio in prostate cancer. Open Access Maced J Med Sci 2019; 7: 1093-6.

27. Shimodaira K, Nakashima J, Nakagami Y, et al. Prognostic Value of Platelet Counts in Patients with Metastatic Prostate Cancer Treated with Endocrine Therapy. Urol J 2020; 17: 42-9. 